\title{
Assessment of Nurses Efficiency Regarding their Performance in the Operating Rooms at Three Teaching Hospitals in Baghdad
}

\author{
Shatha Saadi Mohammad ${ }^{1}$, Wefaqq Mahdi Hadi \\ ${ }^{1}$ Assist. Professor, ${ }^{2}$ Assist. Lecturer College of Nursing, University of Babylon
}

\begin{abstract}
For the purpose of assessing the performance competency of the operating room nurses in Baghdad's hospitals, a descriptive study has been conducted from from 13th of December 2020 through 13 March 2020.

The settings of the performed study were Al-Kindey hospital, Al-Yarmouk Hospital,, Baghdad teaching hospital in Baghdad city, For the study of (50) nurses who have been working definitely in the operating room in these hospitals, a purposeful sample has been selected.

For the study purpose, a special tool has been developed which has been used for data collection. There are three part compromising this tool which are dealing with performance of the nurse, demographic data, and affordability of equipment and supplies in the operating room.

A number of highly qualified experts have been asked to assess the validity of the questionnaire, where they confirmed its validity. As for the questionnaire's legitimacy; it was specified according to the statistically acceptable person correlation coefficient of $(r=92)$.

Data has been analyzed by using analysis of descriptive data (frequencies, percentages) and inferential data analysis (SPSS, 23).

According to the conclusions that have been revealed by study, graduates from universities and institutes had showed a better practices performance than graduates from primary and secondary level.

The study also indicate that scrub nurse practices in some domains were satisfactory (rehearsal of scrub nurses prior to actually entering OR, wearing operating clothes and gloves, draping and sheeting) and inadequate in some fields (practices concerned with surgical preparation, surgical hand scrub, antiseptic and technique and surgical instruments)).

In the study between sexes, a non-significant variation illustrate years of experience in relation to the nurses' training sessions in OR.

The investigation suggested that all nurses ought to circulate strategies to the staff concerning this issue; composed arrangements for the OR practice can be shared to the health-care providers for better execution, and supplies to hospitals such as antiseptic hand scrub strategies that inhibit pathogenic microorganism growth.
\end{abstract}

Keywords: Assessment, nurses' efficiency, operating rooms.

\section{Introduction}

These days' hospitals are furnished with working theater suites with entrance and rehabilitation units supplying several operating theatres, all with anesthetic, implantation, immediate recovery and scrubbing rooms ${ }^{1}$. In surgical services, surgeons, nursing staff, and anesthesia personnel may differ in their conceptions of efficiency, and their motivation to achieve efficient 
processes $^{2}$. All patients and health-care providers are at a higher risk of infection throughout medical procedures due to microorganism exposure. The right attire of surgery can help in post-procedural infection in patients beside the other elements of aseptic strategies, this can be achieved due to decreasing the opportunity of microorganisms entrance into the patients' body ${ }^{3}$. A few components of surgical apparel are as well configured to lower the risk of exposure of health-care professional to potential blood and tissue infections during surgical treatment, including gloves, caps, masks, gowns, protective eyewear and waterproof aprons ${ }^{4}$. Throughout any medical procedure, the most prevalent cause of infection is personals in the operating room. . The main focus is thus on keeping the nursing and medical staff aside from the sterilized gowns and caps that were first revealed to be included in the operating room use ${ }^{5}$. Throughout surgeries, infection in the surgical wound can be precipitated by transferring germs and bacteria from the surgical team's hands for careful surgical scrubbing to lower the amount of bacteria on the skin ${ }^{6}$. Among the main reasons of mortality and morbidity is nosocomial or hospital-acquired infection in the hospitalized patients, hospital-acquired infection development can affected by some elements, and cross-infection from patients to patients can take many routes which will lead to a difficulty in making a link between these variable person and nosocomial infection ${ }^{7}$. Nosocomial infection is significantly correlated the infection at the surgical site. Most of the risk factors for nosocomial infection have involved the behavior of operating room personnel with relation to the practice of contamination by hand hygiene/antisepsis and universal precaution ${ }^{8}$ Nursing staff who work in the theatre can play a great part in the surgical site prevention of infection by undertaking preoperative scrub nurse. In addition, aseptic barriers including sterile gowns and gloves protect sterile areas, isolate clinical sites from pollutants caused by infections and lessen microorganism $>$ s number. ${ }^{9}$

\section{Methodology}

This descriptive research was carried out to assess the nurse's efficiency performance at the operating rooms in three surgical hospitals in Baghdad.

The study started from 13th of December 2020 through 13 March 2020. The sample was non-probability (purposive sample) of 50 nurses was selected from three surgical hospitals in Baghdad and they accepted to participate in the study and taken into consideration: age, gender, and level of education

The data were collected by interviews and constructed with nurse ss efficiency performance at the operating room questionnaire, after getting the permission of Ministry of Health prior to the initiation of the study.

The agreement of participation was obtained from the nurses who were working in the operating room in these hospitals for this present study and they were responding to the interviewing.

Pilot study have evaluated the questionnairess validity and a group of experts to assess the validity. The data were analyzed by applying inferential statistical procedures, percentage, and descriptive statistical frequency. A questionnaire format constructed following enormous review of the available literature and related studies. There are two components of a questionnaire format which include: demographic data for nurses, gender,level of education, and years of experience in hospitals, years of experience in operating room, number of training sessions.

The other part observational questionnaire about the nurses practice in the operating room including scrubbing, equipment and supplies availability, and the nurses usage of the aseptic technique in surgical procedures.

\section{Results}

Table (1): Distribution of demographic characteristics of the nurses

\begin{tabular}{|l|c|c|}
\hline Demographic characteristics of the nurses & F & \% \\
\hline 1-Gender & 39 & 78.0 \\
\hline Male & 11 & 22.0 \\
\hline Female & & \\
\hline
\end{tabular}




\begin{tabular}{|c|c|c|}
\hline Demographic characteristics of the nurses & $\mathbf{F}$ & $\%$ \\
\hline \multicolumn{3}{|l|}{ 2-Marital status } \\
\hline Single & 40 & 80.0 \\
\hline Married & 10 & 20.0 \\
\hline \multicolumn{3}{|l|}{ 3-Educatinoal status } \\
\hline Primary & 9 & 18.0 \\
\hline Secondary & 5 & 10.0 \\
\hline Graduated & 24 & 48.0 \\
\hline Higher Education & 12 & 24.0 \\
\hline \multicolumn{3}{|l|}{ 4-Years of experience in hospital } \\
\hline $1-5$ & 32 & 64.0 \\
\hline $6-10$ & 11 & 22.0 \\
\hline $11-15$ & 6 & 12.0 \\
\hline$>16$ & 1 & 2.0 \\
\hline \multicolumn{3}{|l|}{ 5- Years of experience in OR } \\
\hline $1-5$ & 32 & 64.0 \\
\hline $6-10$ & 14 & 28.0 \\
\hline $11-15$ & 4 & 8.0 \\
\hline \multicolumn{3}{|l|}{ 6- Number of training sessions in OR } \\
\hline 0 & 6 & 12.0 \\
\hline 1 & 1 & 2.0 \\
\hline 2 & 42 & 84.0 \\
\hline 3 & 1 & 2.0 \\
\hline
\end{tabular}

Table (2): Frequency and percentages and mean for nurse's performance

\begin{tabular}{|l|c|c|c|c|c|c|c|c|}
\hline \multirow{2}{*}{ Practice } & \multicolumn{2}{|c|}{ High MS } & \multicolumn{2}{|c|}{ Moderate MS } & \multicolumn{2}{|c|}{ Low MS } & \multicolumn{2}{|c|}{ Total } \\
\cline { 2 - 9 } & $\mathbf{F}$ & $\mathbf{\%}$ & $\mathbf{F}$ & $\mathbf{\%}$ & F & $\%$ & F & $\%$ \\
\hline 1-Scrub nurses preparation before entering the OR & 14 & 82.3 & 0 & 0 & 3 & 17.7 & 17 & 100.0 \\
\hline 2-Surgical hand scrub & 9 & 47 & 2 & 11.7 & 6 & 41.3 & 17 & 100.0 \\
\hline 3-Clothes \& gloves of operation & 7 & 87.5 & 0 & 0 & 1 & 12.5 & 8 & 100.0 \\
\hline 4-Draping \& sheets & 6 & 75 & 1 & 12.5 & 1 & 12.5 & 8 & 100.0 \\
\hline 5-Practice during surgical operation & 17 & 84.5 & 2 & 10.0 & 1 & 5.0 & 20 & 100.0 \\
\hline 6-Antiseptic solution \& surgical instrument & 2 & 50 & 2 & 0 & 2 & 50 & 4 & 100.0 \\
\hline Total & $\mathbf{5 5}$ & $\mathbf{7 2 . 9}$ & $\mathbf{5}$ & $\mathbf{6 . 7 5}$ & $\mathbf{1 4}$ & $\mathbf{2 0 . 2 5}$ & $\mathbf{7 4}$ & $\mathbf{1 0 0 . 0}$ \\
\hline
\end{tabular}


Table (3) Association between sex of the nurses \& their practices in OR
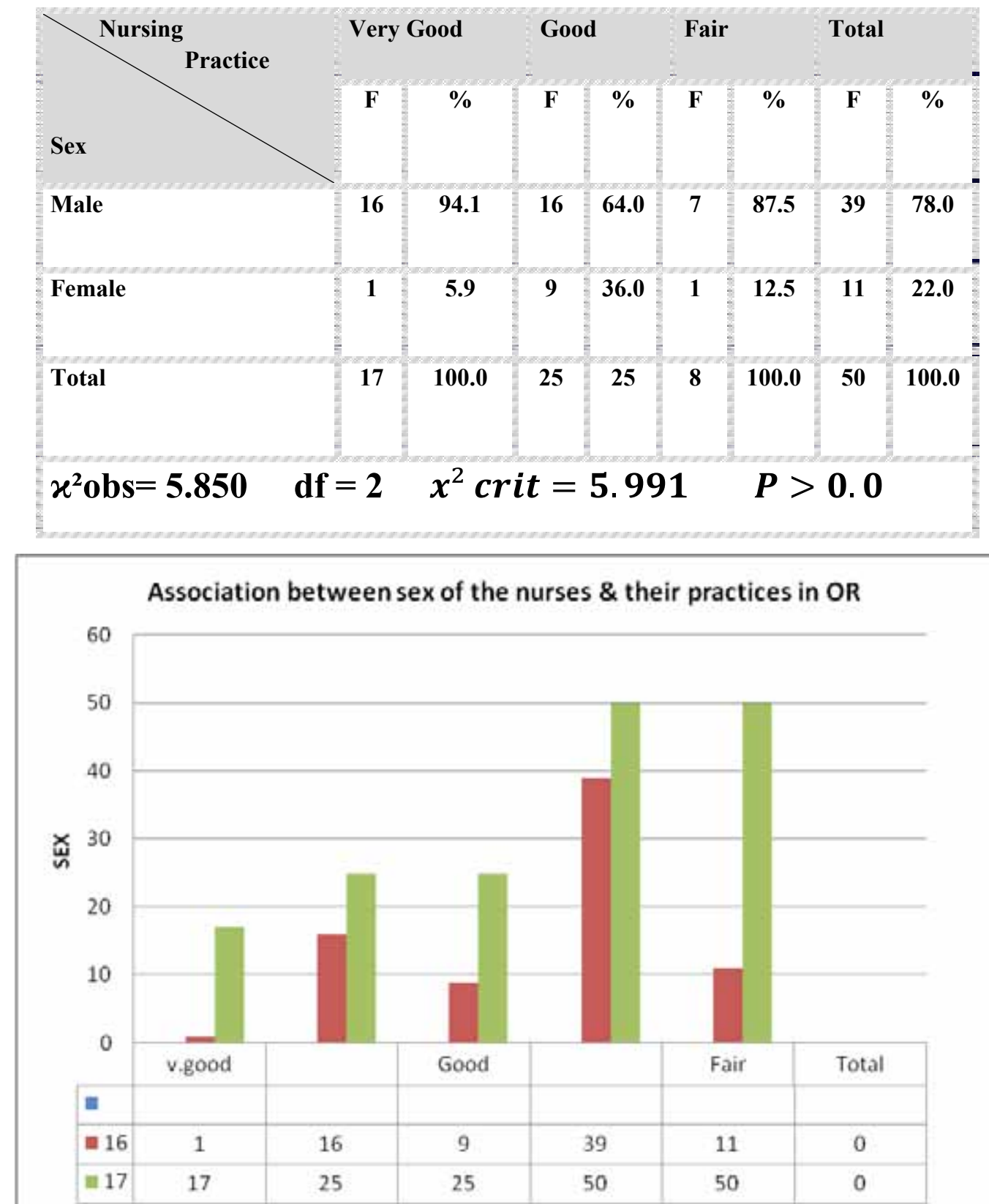

Table (4): Association between educational level of the nurses and their practices in OR

\begin{tabular}{|l|c|c|c|c|c|c|c|c|}
\hline Nursing practice & \multicolumn{2}{|c|}{ Very good } & \multicolumn{2}{c|}{ Good } & \multicolumn{2}{c|}{ Fair } & \multicolumn{2}{c|}{ Total } \\
\hline Level of education & $\mathbf{F}$ & $\mathbf{\%}$ & $\mathbf{F}$ & $\mathbf{\%}$ & $\mathbf{F}$ & $\%$ & F & \% \\
\hline University & 8 & 47.0 & 3 & 12.0 & 1 & 12.5 & 12 & 24.0 \\
\hline Institute & 7 & 41.2 & 12 & 48.0 & 5 & 62.5 & 24 & 48.0 \\
\hline Secondary & 0 & 0 & 5 & 20.0 & 0 & 0 & 5 & 10.0 \\
\hline Primary & 2 & 11.8 & 5 & 20.0 & 2 & 25.0 & 9 & 18.0 \\
\hline Total & $\mathbf{1 7}$ & $\mathbf{1 0 0 . 0}$ & $\mathbf{2 5}$ & $\mathbf{1 0 0 . 0}$ & $\mathbf{8}$ & $\mathbf{1 0 0 . 0}$ & $\mathbf{5 0}$ & $\mathbf{1 0 0 . 0}$ \\
\hline$x^{2}$ obs $=11.863 \mathrm{df}=6 x^{2}$ crit $=12.592 \mathrm{P}>0.05$ & & & & \\
\hline
\end{tabular}


Table (5): Association between nurses practices and their experiences in OR

\begin{tabular}{|l|c|c|c|c|c|c|c|c|}
\hline Nursing practice & \multicolumn{2}{|c|}{ Very good } & \multicolumn{2}{|c|}{ Good } & \multicolumn{2}{c|}{ Fair } & \multicolumn{2}{c|}{ Total } \\
\hline Years of experiences in OR & F & \% & F & \% & F & $\%$ & F & $\%$ \\
\hline $1-5$ & 14 & 82.4 & 12 & 48.0 & 5 & 62.5 & 31 & 62.0 \\
\hline $6-10$ & 2 & 11.8 & 11 & 44.0 & 1 & 12.5 & 12.5 & 28.0 \\
\hline $11-15$ & 1 & 5.9 & 2 & 8.0 & 2 & 25.0 & 25.0 & 10.0 \\
\hline Total & $\mathbf{1 7}$ & $\mathbf{1 0 0 . 0}$ & $\mathbf{2 5}$ & $\mathbf{1 0 0 . 0}$ & $\mathbf{8}$ & $\mathbf{1 0 0 . 0}$ & $\mathbf{1 0 0 . 0}$ & $\mathbf{1 0 0 . 0}$ \\
\hline$x^{2}$ obs $=8.687 \mathrm{df}=4 \boldsymbol{x}^{2}$ crit $=9.488 \mathrm{P}>0.05$ &
\end{tabular}

\section{Discussion}

Working in a team is essential for successful surgery, even though there are presently no team cohesion indicators to direct training, to analyses the effect of group work on consequences, the researcher will report the first stages in the development of an observational teamwork evaluation and preliminary findings. ${ }^{10}$ In this study we found that the majority of the nurses were males, (78\%) graduated, (48\%) single, $(80 \%)$ having (15) years of experience in hospital and OR (64\%), and they had enrolled in (2) time training sessions (84\%) (Table 1) These results coincided with (Shabnam 2006), findings in his study of the sample consisted from 50 nurses who worked actually in OR and most of them were males, single but their practices were found to be unsatisfactory in OR. It has been realized that the scrub nurses in the examination of table (2) did not perform their practice of preparation before entering OR in many sub-terms of this preparation although there was a high MS in the domains of $(1,3,4,5)$ related to the performs of practice for preparation before entering OR. ${ }^{11}$ Conducted a stated that reported scrub nurses should take bath daily with soap that contains an antibacterial agent, which can reduce shedding. Also, the present study realized that in sub-items in this domain that $(20 \%)$ of those scrub male nurses did not shave their face. ${ }^{12}$ Stated that hair face could be considered a major source of staphylococci. Furthermore, the nurses had put the mask before surgical hand scrub (100\%) and they do not touch surgical instruments by hand after the surgical hand scrub (98\%). Relative to their practice in the OR, they also observed adequate practice, which was presented through using a sterilizing brush in the hand surgical scrubbing, and they used it between fingers $(88 \%)$. On the other side, the nurses did not use woods, nail cleaner under nails, and antiseptics solution at the beginning because the supplies were not available in the hospitals. As noted by 13 in his study that the (WHO) The hospital embraced hand hygiene protocols which its research carried out on a team that made comprehensive workflow diagrams for prevalent patient care duties identifying evidence of hand washing. Three months later, health care staff have been equipped to evaluate one another and forwarded relevant information to the Department of Infection Prevention and Control Following the launch of the program, conformance expanded from $60 \%-70 \%$ to $97 \%$ and remains to be maintained at that point. Adequate performance of practice was presented through wearing the gown and gloves and raising the sterilizing hand above hips. In addition, proper surgical attire reduces the danger of clients infection with post-procedures by lowering micro-organisms entering locations of the client's body throughout the surgery. ${ }^{14}$ Revealed that appropriate surgical apparel can diminish the risk of post-procedure infections in clients by decreasing microorganisms that enter the area of the client's body during the procedure. Throughout data analysis, the study findings had confirmed that all scrub nurses, regardless of their sex has performed adequate practice in the operating room. (Table 3),(Figure 1). Regarding their educational level university graduates $(47.0 \%)$ and institute graduates $(48 \%)$ presented better performance of practice than secondary and primary school graduates. (Table 4). ${ }^{15}$ Reported in his study that staff nurses had performed adequate practices than practical ones in OR. Concerning the experiences in OR, the study shows that the scrub nurses' practice was not influenced with years of experience because the correlation between their practices and years of experience was found to be not significant for all domains, but the scrub nurses who had (1-5) years of experience had high -level practice than others. (Table 5) The findings shows that beginner scrub training is influenced externally by its perioperative 
cultural environment and the encourage of senior employees. To sum up, it is extremely important to highlight the role of perioperative cultural surroundings and the support of the senior staff to nurses regarding their novice scrub learning. As senior scrub staff dictated their educating to novices according to their attitudes.

Financial Disclosure: There is no financial disclosure.

Conflict of Interest: None to declare.

Ethical Clearance: All experimental protocols were approved under the College of Nursing and all experiments were carried out in accordance with approved guidelines.

\section{References}

1. Cuschieri A. Human reliability assessment in surgery--a new approach for improving surgical performance and clinical outcome. An nn R Coll Surg Engl. 2000; 82(2): 83-87.

2. Arakelian E.How operating room efficiency is understood in a surgical team: a qualitative study Int J Qual Health Care. 2011; 23(1):100-6.

3. Yule S, Flin R, Paterson-Brown S, Maran N. Nontechnical skills for surgeons in the operating room: A review of the literature. Surgery. 2006; 139: 140149.

4. Jacqueline RN, Yan X. The Operating Room Charge Nurse: Coordinator and Communicator. J Am Med Inform Assoc. 2002; 9(6): s70-s74.

5. Hong C. Assessing the Performance of Operating
Rooms: What to Measureand Why? Proceedings of Singapore Healthcare. 2011;20: 2.

6. Stobinski JX. Perioperative nursing competency. AORN J. 2008; 88(3):417-8, 421-36.

7. S Yule. Non-technical skills for surgeons in the operating room: A review of the literature. Surgery. 2006;139(2).

8. Lingard L. Team communications in the operating room: talk patterns, sites of tension, and implications for novices. Acad Med. 2002; 77(3):232-7.

9. Booij LH, van Leeuwen E. Teamwork and the legal and ethical responsibility of the anaesthetist. Curr Opin Anaesthesiol. 2008; 21(2):178-82.

10. Macario A. Are your hospital operating rooms "efficient"? A scoring system with eight performance indicators. Anesthesiology. 2006; 105(2): 237-40.

11. Park K, Dickerson C. Can efficient supply management in the operating room save millions? Curr Opin Anaesthesiol. 2009; 22(2):242-8.

12. Leach LS, Myrtle RC, Weaver FA, Dasu S. Assessing the performance of surgical teams. Health Care Manage Rev. 2009; 34(1):29-41.

13. Elaine L. Monitoring hand hygiene: Meaningless, harmful, or helpful? 2013; 15: S42-S45.

14. Bleyl JU, Heller AR. Standard operating procedures and operating room management: Improvement of patient safety and the efficiency of processes. Wien Med Wochenschr. 2008; 158(21-22):595-602.

15. Booij LH. Conflicts in the operating theatre. Curr Opin Anaesthesiol. 2007; 20(2):152-6. 